\title{
AtaT Improves the Stability of Pore-Forming Protein EspB by Acetylating Lysine 206 to Enhance Strain Virulence
}

\author{
Zhili He, Tao Li, Jianxin Wang, Deyan Luo, Nianzhi Ning, Zhan Li, Fanghong Chen and \\ Hui Wang*
}

State Key Laboratory of Pathogens and Biosecurity, Beijing Institute of Microbiology and Epidemiology, Beijing, China

A novel type II toxin of toxin-antitoxin systems (TAs), Gcn5-related N-acetyltransferase (GNAT) family, was reported recently. GNAT toxins are mainly present in pathogenic species, but studies of their involvement in pathogenicity are rare. This study discovered that the GANT toxin AtaT in enterohemorrhagic Escherichia coli (EHEC) can significantly

OPEN ACCESS

Edited by:

Frank Ebel,

Ludwig Maximilian University of Munich, Germany

Reviewed by:

Karl Kuchler

Medical University of Vienna, Austria Yoshihiro Yamaguchi

Osaka City University, Japan

${ }^{*}$ Correspondence:

Hui Wang

geno0109@vip.sina.com wanghui_dyx@hotmail.com

Specialty section: This article was submitted to

Infectious Diseases,

a section of the journal

Frontiers in Microbiology

Received: 08 November 2020 Accepted: 27 January 2021

Published: 01 March 2021

Citation:

He Z, Li T, Wang J, Luo D, Ning N, Li Z, Chen F and Wang $H$ (2021) AtaT Improves the Stability of Pore-Forming Protein EspB by Acetylating Lysine 206 to Enhance

Strain Virulence.

Front. Microbiol. 12:627141. doi: 10.3389/fmicb.2021.627141 enhance strain pathogenicity. First, we detected the virulence of $\Delta a t a T$ and $\Delta a t a R$ in cell and animal models. In the absence of ataT, strains showed a lower adhesion number, and host cells presented weaker attaching and effacing lesions, inflammatory response, and pathological injury. Next, we screened the acetylation substrate of AtaT to understand the underlying mechanism. Results showed that $E$. coli pore-forming protein EspB, which acts as a translocon in type III secretion system (T3SS) in strains, can be acetylated specifically by AtaT. The acetylation of K206 in EspB increases protein stability and maintains the efficiency of effectors translocating into host cells to cause close adhesion and tissue damage.

Keywords: Gcn5-related $\mathrm{N}$-acetyltransferase, AtaT, enterohemorrhagic Escherichia coli, EspB, virulence

\section{INTRODUCTION}

Toxin-antitoxin systems (TAs) are widely present on prokaryote plasmids and chromosomes (Gerdes et al., 2005). These systems consist of two co-expression genes, encoding stable toxin and sensitive antitoxin. TAs were initially discovered as plasmid maintenance modules (Ogura and Hiraga, 1983; Gerdes et al., 1986; Bravo et al., 1987). Unstable antitoxins in plasmid-free cells cannot be supplemented, leaving the toxin free to eliminate or arrest the growth of these cells, which results in the maintenance of plasmids in the bacterial population. The advent of genome sequencing has further exposed the abundant TAs encoded on prokaryotes' chromosomes (Van Melderen and Saavedra De Bast, 2009; Riffaud et al., 2020). Chromosome TA modules do not maintain plasmid stability. Their functions are associated with different metabolic processes and growth controls to cope with adverse environments (Yamaguchi and Inouye, 2011; LobatoMarquez et al., 2016; Rocker and Meinhart, 2016; Harms et al., 2018; Paul et al., 2019; Walling and Butler, 2019). However, there are heated debates about the participation of chromosome TAs in environmental stress (Tsilibaris et al., 2007; Goormaghtigh et al., 2018a,b; Holden and Errington, 2018; Fraikin et al., 2020), and their role in organisms is more complicated and confusing than ever. 
There are six different types of TAs according to the mechanism of toxin and antitoxin interaction, of which type II TAs research is the most in-depth. In recent years, scientists have discovered a new type II toxin that belongs to the Gcn5-related $N$-acetyltransferase (GNAT) (Cheverton et al., 2016; Jurenas et al., 2017; Qian et al., 2018, 2019; Wilcox et al., 2018). GNAT toxin blocks protein translation by acetylating the amino group of charged tRNAs, thus preventing tRNA from participating in peptidyl ribosomal transferase (Yeo, 2018). Reports suggest that GNAT toxins are mainly present in pathogenic species. Most GNAT toxins are distributed in the genomes of Salmonella enterica and Klebsiella pneumoniae (Xie et al., 2018); other major species carrying GNAT toxins are Escherichia coli and Mycobacterium tuberculosis (Qian et al., 2018). To date, the functions of several GNAT toxins in pathogenic species have been analyzed. KacT toxin contributes to antibiotic tolerance in K. pneumoniae (Qian et al., 2018); the expression of TacT in Salmonella typhimurium promotes the strain persistence in macrophages (Cheverton et al., 2016; Rycroft et al., 2018); and GmvT in Shigella stabilizes pathogenicity island-harboring pINV plasmid (McVicker and Tang, 2016). However, the function of GNAT toxins in other species is still largely unknown.

Enterohemorrhagic E. coli (EHEC) is a pathogenic Gramnegative bacterium. It can cause severe hemorrhagic colitis and hemolytic uremic syndrome in infected human bodies. It is also highly contagious and can lead to pandemic outbreaks. Epidemiological control is a challenge because it has a low infectious dose and sophisticated pathogenic mechanisms (Cameron et al., 2018; Lang et al., 2018). AtaT, a recently discovered GNAT toxin in E. coli O157:H7, uses acetyl-coenzyme A to block translation initiation by specifically acetylating

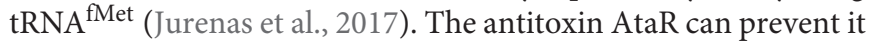
from forming an active dimer to neutralize its toxicity (Yashiro et al., 2019). Jurënas and his colleagues explored the function of this TAs and concluded that the AtaRT system might be involved in anti-addiction and that Ata $\mathrm{T}$ is unlikely to participate in persistence (Van Melderen et al., 2018). A quick BLAST search shows that AtaRT is also widely distributed in E. coli O157:H7 Sakai, Xuzhou21, and many other pathogenic strains including E. coli O55:H7 RM12579, CB9615, E. coli APEC O78, and E. coli NA114. As a GNAT toxin mainly distributed in pathogenic species, it is unclear whether ataT is involved in strains' pathogenic processes.

In this study, we revealed that GNAT toxin AtaT enhances the virulence of strains by maintaining the stability of pore-forming protein EspB through acetylation.

\section{MATERIALS AND METHODS}

\section{Ethics Statement}

This study was carried out according to the recommendations set out in the Guide for the Beijing Institute of Microbiology and Epidemiology Animal Care and Use Committee (2016-0511-05). The protocol was approved by the Institutional Ethics Review Committee of Beijing Institute of Microbiology and Epidemiology, China. Mice were purchased from Vital River
Laboratory Animal Technology, Beijing, China (permit number: 2016-0006). The female BALB/c mice (14-16 g) were maintained on either a regular diet (standard mice feed and $12 \mathrm{~h}$ for light/dark alternate). All animal work was carried out strictly under the approved guidelines, and all efforts were made to minimize suffering.

\section{Bacterial Strains and Plasmids}

All of the strains and plasmids used in this study are listed in Supplementary Table 1 . Bacteria were grown with shaking at $37^{\circ} \mathrm{C}$ in lysogenic broth (LB) culture or Dulbecco's modified Eagle's medium (DMEM) media. Ampicillin (100 $\mu \mathrm{g} / \mathrm{ml})$, chloramphenicol $(25 \mu \mathrm{g} / \mathrm{ml})$, kanamycin $(50 \mu \mathrm{g} / \mathrm{ml})$, and apramycin $(60 \mu \mathrm{g} / \mathrm{ml})$ were used in this study. Wherever indicated, $0.2 \%$ L-arabinose or $0.1 \mathrm{mM}$ of isopropyl $\beta$-D-1thiogalactopyranoside (IPTG) was used as an inducer.

\section{Construction of Mutant Strain}

Mutant strains of EHEC were constructed using the $\lambda$ Red recombineering gene deletion technology. The kanamycinresistant gene kan (flanked by flippase recognition target FRT sites) flanked by homologous sequences of ataT was amplified by PCR. PCR fragments were analyzed by agarose gel electrophoresis on $1 \%$ (wt/vol) agarose gels for $30 \mathrm{~min}$. PCR fragments were purified using the PCR product purified kit (Transgen Biotech). P8 strain (EHEC harboring plasmid pKD46) was grown to an optical density at $600 \mathrm{~nm}$ (OD600) of $\sim 0.6$ at $30^{\circ} \mathrm{C}$ and washed three times with water and glycerol [10\% (vol/vol)]. Two hundred nanograms of PCR fragments was electroporated into P8 (BioRad MicroPulser Electroporator). Cells were then recovered in $0.7 \mathrm{ml}$ of LB for $1 \mathrm{~h}$, plated on LB plus agar (kanamycin), and incubated overnight at $30^{\circ} \mathrm{C}$. The $\Delta$ ataT strain carrying the kanaresistance gene ( $\triangle a t a T: k a n a)$ was obtained. $\Delta a t a T: k a n a$ was streaked repeatedly on antibiotic plates at $42^{\circ} \mathrm{C}$ to cure the strains of plasmid pKD46. Then the plasmid pFLP2 was electroporated into $\triangle$ ata $T$ :kana to remove the kanamycin-resistant gene. pFLP2 was cured via culture in LB with $6 \%$ sucrose $(w / v)$. The $\triangle a t a R$ strain was engineered as follows.

\section{Cellular Infection}

The day before infection, approximately $1 \times 10^{5}$ of HT29 (human colon cancer cells) were seeded in DMEM (10\% fetal bovine serum, \#10099141C, Gibco) on six-well plates or coverslips without antibiotics. Overnight culture of strains EHEC wild-type (WT), $\triangle a t a T$, and $\triangle a t a R$ were transferred into fresh DMEM medium at a ratio of 1:1,000. When OD600 $\mathrm{nm}=0.6$, cells were infected at multiplicity of infection $(\mathrm{MOI})=10$ for different periods of time. Phosphate-buffered saline (PBS)treated cells were used as a control. Next, we processed the different batches of cells in the following steps: (1) we removed supernatants and treated the cells with $0.25 \%$ trypsin (\#25200072, Gibco), and the bacteria adhering to the cells were collected. Total RNA was isolated (\#ER501-01, Trans, Beijing, China), and cDNA was generated (\#AT341-02, Trans, Beijing, China) by reverse transcription using RNA as a template. The levels of mRNA were analyzed by quantitative real-time PCR (\#AQ131-03, Trans, Beijing, China). (2) We removed supernatants, and the cells were 
washed three times with PBS. After being treated with $0.25 \%$ trypsin for $2 \mathrm{~min}$ and $0.025 \%$ Triton X-100 for $10 \mathrm{~min}$, the cell suspension per well was fixed to a volume of $1 \mathrm{ml}$ by PBS. The gradient-diluted cell lysates were inoculated on agar plates, and bacterial colonies were counted. (3) After re-culturing in DMEM containing antibiotics for another 6-8 h, the level of IL-8 in the supernatant was detected by ELISA (\#EHC008.96, NeoBioscience, Beijing, China). (4) HT-29 cells on the coverslips were fixed and stained with antibody to E. coli O157 (\#ab156617, Abcam), Alexa Fluor 488 goat anti-mouse IgG (\#A-11029, Invitrogen), Alexa Fluor 647 phalloidin (\#A30107, Invitrogen), and DAPI (\#C0065, Solarbio, Beijing, China); and the slices were subjected to fluorescence microscopy (Zeiss LSM880).

\section{Mice Infection}

Fifteen female BALB/c mice (pre-treated with $5 \mathrm{~g} / \mathrm{L}$ of streptomycin for 3 days) per group were orally gavaged with EHEC WT, $\triangle a t a T$, or $\triangle a t a R\left[1 \times 10^{9}\right.$ colony-forming units (CFU)]. At the same time, PBS was used as a negative control. Feces from five mice were randomly taken from each group in indicated days, and the bacterial shedding was counted on Sorbitol-MacConkey agar plates until the 23rd day. On the fourth day of infection, blood was collected from five randomly selected mice in each group. We detected mice serum keratinocytederived cytokine (KC) concentration using a mouse KC kit (EMC104.96, NeoBioscience, China). We isolated the colon; then fixed, sliced, and stained it; and analyzed pathological lesions.

\section{Protein Expression and Purification}

Escherichia coli BL21(DE3) carrying the pETDuet-1-EspB plasmid, expressing EspB (His) 6 , was grown to the logarithmic phase at $37^{\circ} \mathrm{C}$. IPTG was added to make the final concentration $0.1 \mathrm{mM}$ and further cultured for $16 \mathrm{~h}$ at $19^{\circ} \mathrm{C}$. Strains were centrifugated at $4^{\circ} \mathrm{C}$ and disrupted by ultrasonic method. After centrifugation, the sediment was resuspended in urea buffer $(8 \mathrm{M}$ of urea, $100 \mathrm{mM}$ of Tris- $\mathrm{HCl}, \mathrm{pH} \mathrm{8.0)}$ overnight and renatured by step-by-step dialysis. AtaT (His) 6 was purified from E. coli BL21(DE3) harboring pETDuet-1-AtaRT. The complex of AtaRAtaT (His) 6 was captured by Ni-NTA resin for the first time. After dissociation of AtaR from the complex in $5 \mathrm{M}$ of guanidine$\mathrm{HCl}$, AtaT (His) 6 was recaptured by Ni-NTA resin and renatured. Fractions containing purified AtaT (His) 6 or EspB (His) 6 were selected based on sodium dodecyl sulfate-polyacrylamide gel electrophoresis (SDS-PAGE) analysis.

\section{Acetylation Assays in vivo and in vitro}

The acetylation in vivo assay steps were as follows. Vectors pETDut1-espB(his) 6 and pBAD33-ataT were co-expressed in E. coli BL21 (DE3), while pETDut1-espB was expressed alone as a control. IPTG or L-arabinose were added at $1 \mathrm{~h}$ to induce the expression of EspB or AtaT, and the same level of EspB (samples of co-expressed strains were condensed about five times) was collected at $6 \mathrm{~h}$. The modification levels of EspB were detected by acetylated antibody ( $\alpha$-Acetyl) (\#9441, CST). Histidine antibody (anti-His) (\#12698, CST) was used as a control. Other adhesion proteins $\left[\operatorname{EspA}(\text { his })_{6}, \operatorname{LpfA}(\text { his })_{6}, \operatorname{Tccp}(\text { his })_{6}, \operatorname{Intimin}(\text { his })_{6}\right.$, and
$\operatorname{Tir}(\text { his })_{6}$ ] followed the same experimental procedures to detect the level of acetylation.

The in vitro acetylation reaction was performed at $37^{\circ} \mathrm{C}$ for $6 \mathrm{~h}$ by adding $10 \mu \mathrm{g}$ of AtaT, $4 \mu \mathrm{g}$ of EspB, and $0.2 \mathrm{mM}$ of acetyl-CoA in a volume of $50 \mu \mathrm{l}$ to produce the EspB-Ac (Ren et al., 2016), while EspB incubated alone as a control.

\section{Degradation of EspB in vitro}

Dulbecco's modified Eagle's medium with $1 \mathrm{~g} / \mathrm{L}$ glucose was inoculated 1:100 with overnight cultures of EHEC allowed to grow in $5 \% \mathrm{CO}_{2}$ at $37^{\circ} \mathrm{C}$. After $16 \mathrm{~h}$ of growth, the supernatant was collected and filtered through a $0.22-\mu \mathrm{m}$-poresize filter unit (Millipore) to concentrate 50-fold to produce the endogenous protease of EHEC (Cameron et al., 2018). Five hundred nanogram of EspB or EspB-Ac (from acetylation assays in vitro) was exposed to $150 \mu \mathrm{l}$ of concentrated protease and incubated at $37^{\circ} \mathrm{C}$ for $5 \mathrm{~h}$. The same volume of mixtures was collected every hour, and protein levels with histidine antibody (anti-His) detected.

\section{Degradation of EspB in vivo}

The stability of EspB (His) 6 and its derivative mutants was detected via in vivo degradation experiments (Sang et al., 2016). Plasmid harboring esp $B$ (his) $)_{6}$ or its derivatives were induced by $0.1 \mathrm{mM}$ of IPTG for $60 \mathrm{~min}$. Translation was blocked with $100 \mu \mathrm{g} / \mathrm{ml}$ of spectinomycin, and samples were collected every $10 \mathrm{~min}$. The EspB levels were detected with histidine antibody (anti-His), while Dnak levels were detected as a control.

\section{Translocation Assay}

HeLa cells were infected with EHEC WT and mutants (harboring the Tir-TEM1 vector, MOI = 10). After $30 \mathrm{~min}$, IPTG was added and incubated for another $4 \mathrm{~h}$. Cell monolayers were washed with Hank's balanced salt solution (HBSS) three times and covered with CCF2/AM solution (\#K1023, Invitrogen) for $1 \mathrm{~h}$ in darkness at room temperature. The CCF2/AM solution was washed off with HBSS, and cells were observed under a confocal fluorescence microscope (Dichroic mirror, Zeiss LSM880). After being excited by $405 \mathrm{~nm}$, the ratio of blue fluorescence $(460 \mathrm{~nm})$ to green fluorescence $(530 \mathrm{~nm})$ was collected.

\section{RESULTS}

\section{AtaT Enhanced the Adhesion of Enterohemorrhagic Escherichia coli and Inflammatory Response of Host Cells}

Since ataRT is mainly distributed in pathogenic intestinal bacteria, we first explored the relationship between AtaRT and pathogenicity. We used the host cells of EHEC, HT-29 cells, infected with exponential-phase EHEC for hours, and the adherent bacteria were collected while EHEC was grown without cells as a control. Isolated total RNA and real-time qPCR data were analyzed. As shown in Figure 1A, at post-infection 2-6 h, ata $T$ and $a t a R$ were upregulated, and their expression decreased at $8 \mathrm{~h}$. This was consistent with ler, which encodes Ler protein 


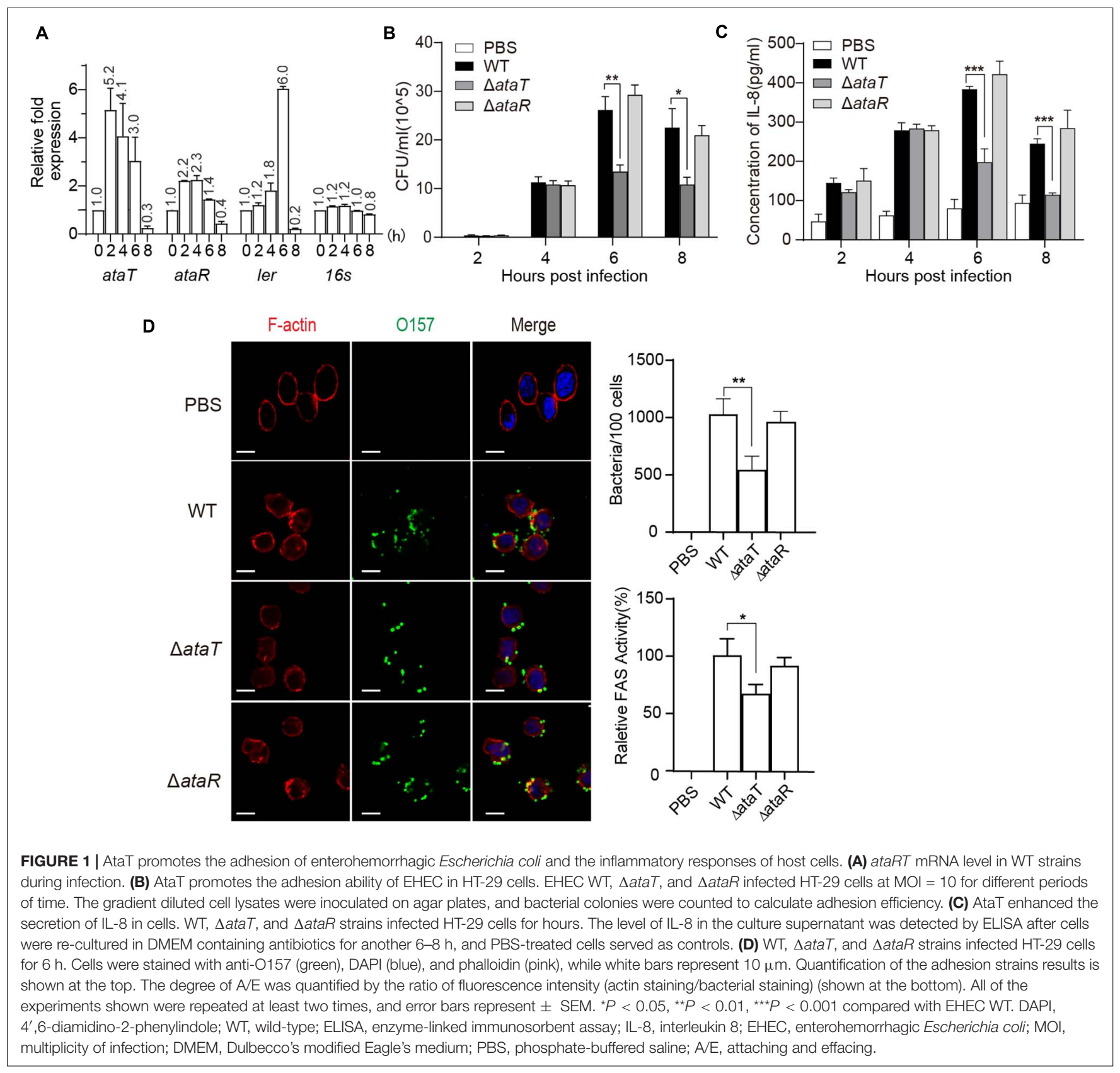

(LEE-encoded regulator) and is one of the essential regulatory molecules during EHEC adhesion (Alsharif et al., 2015).

Then, we assessed several pathogenicity indicators in EHEC, including Shiga toxin production ability and adhesion ability. The growth curves of the gene-deleted mutants $\Delta a t a T$ and $\triangle a t a R$ are shown in Supplementary Figure 1B. The $\Delta a t a T$ growth conditions were consistent with EHEC WT, while $\triangle a t a R$ exhibited growth inhibition because the repression of the toxin gene ataT was released (Supplementary Figure 1C). We next detected the levels of Shiga toxin produced by these strains when infecting HT-29 cells for $6 \mathrm{~h}$, and no significant difference was observed (Supplementary Figure 2). However, in the adhesion test experiment, we found an interesting phenomenon. The bacterial adhesion number of $\triangle a t a T$ was reduced by $50 \%$ compared with WT at 6 and $8 \mathrm{~h}$, and the adhesion of $\triangle a t a R$ was consistent with WT (Figure 1B). This means that the toxin may be involved in the adhesion of the strain. Cytokines are responsible for regulating the inflammatory response. More bacterial adhesion will lead to more severe inflammation. We therefore collected the supernatants of infected cells to detect the levels of cell inflammatory factor IL-8. As expected, WT and $\triangle a t a R$ groups possessed higher inflammatory response than the $\triangle$ ata $T$-infected group at 6 and $8 \mathrm{~h}$ (Figure 1C). Relative fluorescence actin staining (FAS) activity (Luo and Donnenberg, 2006) under the microscope also showed that the attaching and effacing $(\mathrm{A} / \mathrm{E})$ lesions of cells in the $\Delta a t a T$-infected group 
were significantly less than those in the WT and $\Delta a t a R$ groups (Figure 1D). All of the results demonstrated that type II toxins AtaT can enhance EHEC's adhesion ability and cause several inflammatory responses.

\section{The $\Delta$ ataT Attenuated Bacterial Localization and Inflammation in the Colon}

As a molecule that aggravates cell infection, we wanted to know the role of AtaRT in mouse models. BALB/c female mice were randomly divided into four groups with 15 mice in each group. They were given intragastric administration of $1.0 \times 10^{9} \mathrm{CFU}$ of WT, $\triangle a t a T$, or $\triangle a t a R$. We detected the amount of strain shedding in the feces and tested the colonization of different strains. Feces suspensions were plated on Sorbitol-MacConkey agar plates, and bacterial counts were plotted as the average CFU per gram of feces. We observed that the strain excretion in group $\triangle a t a T$ was markedly lower than in the other two groups from days 3 to 23 (Figure 2A). This test confirmed that AtaT maintained the strong intestinal colonization ability of the strain. We detected the $\mathrm{KC}$, a functional homolog of human IL-8 (Li et al., 2017), levels in the serum on the fourth day. As depicted in Figure 2B, compared with the WT-infected group, deletion of ataT significantly decreased $\mathrm{KC}$ levels by approximately $50 \%$, while $\triangle a t a R$ could replenish KC levels. We also took out the colon and fixed the section to evaluate the pathological lesions on this day. There were distinct pathology lesions in WT or $\Delta$ ataR-infected mice, such as the intestinal cavity showing shedding epithelium, infiltration of inflammatory cells in the acinus lamina propria acinar absence atrophy, and local edema. Only mild inflammation was observed in $\Delta a t a T$ infected mice (Figure 2C). At the same time, we set the scoring criteria and scored the slices. The colon injury severity scores clearly showed that colon inflammation of $\Delta a t a T$-infected mice was much milder $(P<0.01)$, but that the colon remained severely inflamed after infection with EHEC WT or $\triangle a t a R$ (Figure 2D), indicating that less $\Delta a t a T$ colonized colon and caused slighter pathological damage.

\section{AtaT Promotes the Stability of EspB by Acetylation to Enhance the Translocation of Effectors Into Host Cells}

We then explored the molecular mechanism of AtaT increasing EHEC colonization. AtaT has been identified as a member of the acetyltransferase family. We co-expressed various adhesion proteins with AtaT or without and detected the acetylation levels of these proteins. As AtaT affects bacterial growth (Supplementary Figure 3), samples of co-expression groups
A

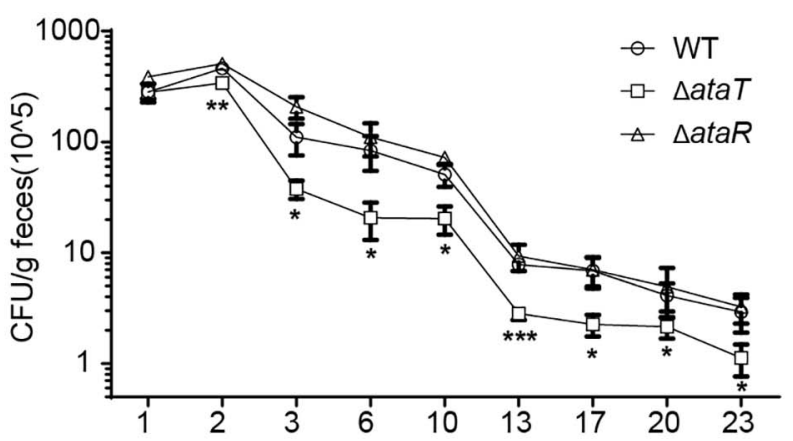

C

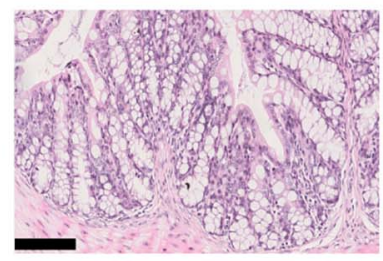

PBS

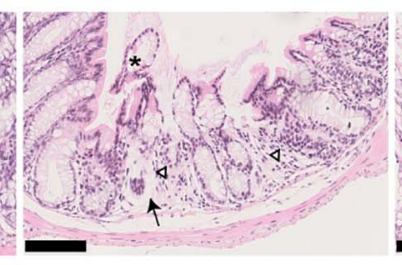

WT
B

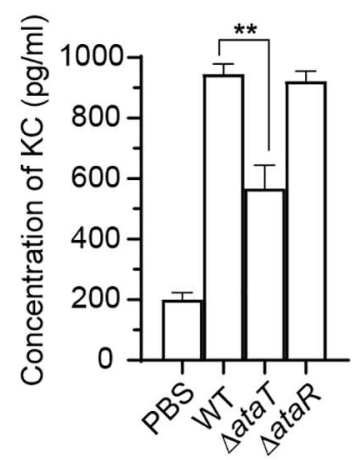

D

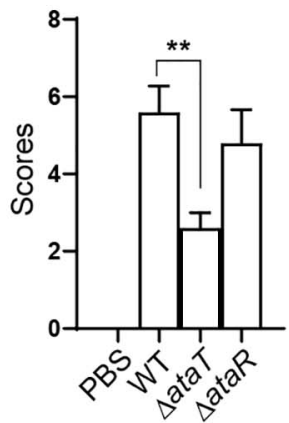

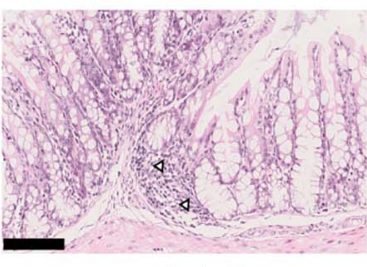

$\Delta$ ataT

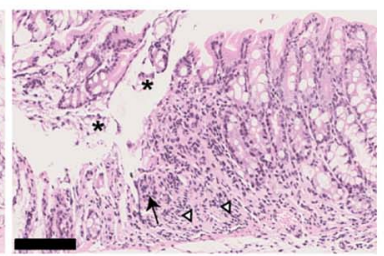

$\Delta a t a R$

FIGURE 2 | AtaT increases colonization of EHEC and causes severe inflammation in the colon. (A) Magnitude of fecal shedding of different strains. WT, $\triangle$ ataT, and $\triangle a t a R$ strains infected streptomycin-treated BALB/c mice for up to 23 days. Feces suspensions were plated on Sorbitol-MacConkey agar plates, and bacterial counts are plotted as the average CFU per gram of feces. (B) AtaT increases the level of serum KC levels. ELISA detected the levels of KC on the fourth day and PBS-gavaged mice served as controls. (C) AtaT increases colon injury in infected mice. The colons of mice infected for 4 days in each group were sectioned, fixed, and stained. Inflammatory cells (triangle), exfoliated epithelium (asterisk), and acinar atrophy with local edema (arrow) were marked, and bars represent 100 $\mu \mathrm{m}$. (D) The colon injury severity score. The degree of colonic pathological injury ranged from the slightest to the most severe, with a score of $0-10$. Three to five fields of vision were selected for each slide. Error bars represent \pm SEM. ${ }^{\star} P<0.05$, ${ }^{\star \star} P<0.01,{ }^{\star \star \star} P<0.001$ compared with EHEC WT. All of the experiments shown were repeated at least two times. WT, wild-type; ELISA, enzyme-linked immunosorbent assay; CFU, colony-forming units; KC, keratinocyte-derived cytokine; EHEC, enterohemorrhagic Escherichia coli; PBS, phosphate-buffered saline. 
needed to be concentrated about fivefold to reach the same protein level as the controls. As shown in Figure $\mathbf{3 A}$, the acetylation level of EspB with AtaT co-expression increased eightfold compared with expression alone. However, other adhesion proteins, such as Tir and Intimin, could not be acetylated, regardless of whether AtaT was present. These results showed that EspB is the specific substrate of AtaT; this may explain why AtaT can maintain the strain intestinal colonization. In addition, we performed degradation systems for EspB in vitro and in vivo. First, purified EspB and EspB-Ac (treated with AtaT and acetyl-CoA) were exposed to endogenous protease EspP (E. coli-secreted protein P) from EHEC (Cameron et al., 2018). It was observed the half-life of purified EspB increased 2.3-fold after being treated with AtaT in vitro (Figure 3B).
Subsequently, compared with that of WT, the half-life of EspB in $\triangle a t a T$ was shortened to nearly $50 \%$. The stability of EspB can be restored by replenishing ataT in the background of $\triangle a t a R$ in vivo (Figure 3C). The above results suggested that AtaT maintains the stability of EspB in vivo and in vitro.

EspB is a translocon protein of the type III secretion system (T3SS) and forms the T3SS pore in host cells. As an adhesion protein, Tir is the first effector to be injected into host cells (Mills et al., 2013). If the stability of EspB changes, the translocation of Tir through T3SS is also affected. Therefore, we tested Tir translocation efficiency in mutants and WT strains by using the TEM-1- $\beta$-lactamase reporter. Strains harboring the Tir-TEM1 or TEM1 vector infected HeLa cells for $6 \mathrm{~h}$. The relative translocation efficiency of TEM-1 was represented by

A
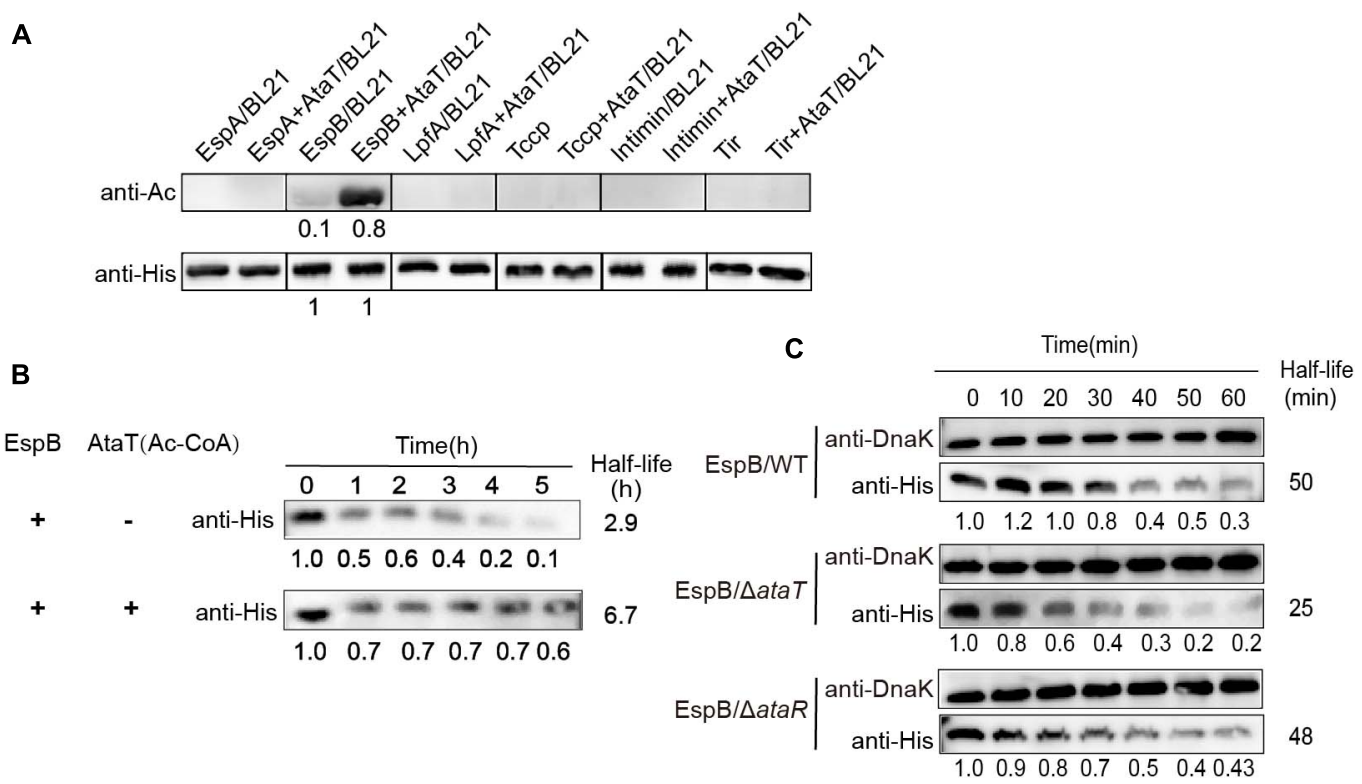

D
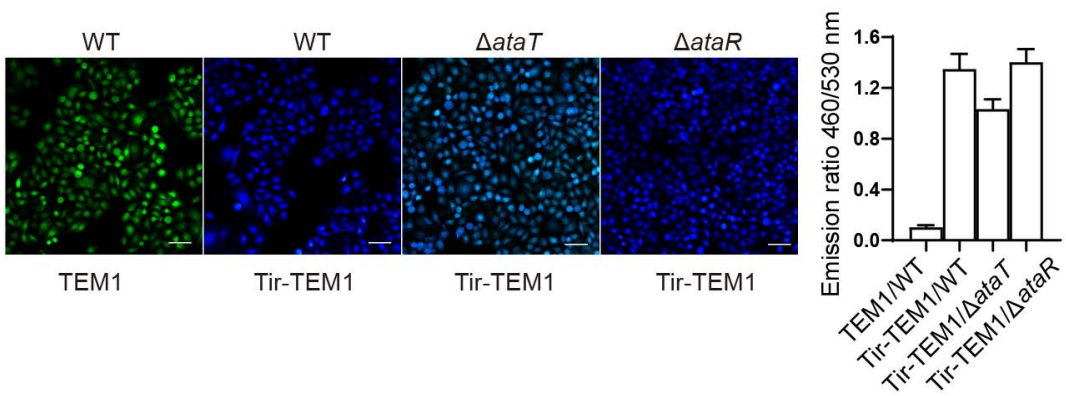

FIGURE 3 | EspB promotes its stability through acetylation by AtaT to keep Tir translocate into host cells. (A) EspB is the AtaT-specific acetylation modification target in vivo. Detection of the acetylation level of adhesion proteins $\left[\mathrm{EspA}(\text { his })_{6}, \mathrm{EspB}(\mathrm{his})_{6}\right.$, LpfA(his)6, Tccp(his)6, Intimin(his), and Tir(his)6], which are expressed alone or co-expressed with AtaT in Escherichia coli strain BL21. Acetylation levels were detected by the anti-acetyl lysine antibody (anti-Ac) and the anti-His antibody (anti-His) was used as a control. (B) Acetylation of EspB enhances protein stability in vitro. Purified 6xHis-tagged EspB was exposed to T3SS enzyme with or without purified AtaT and Ac-CoA for hours. Protein levels were detected by anti-His antibody. (C) Stability of EspB in EHEC WT, $\Delta$ ataT, and $\Delta$ ataR strain. Strains harboring EspB expression plasmid was induced by IPTG for $1 \mathrm{~h}$. Spectinomycin was used to terminate protein synthesis at different points, and strains were collected. The level of EspB was detected by anti-His antibody, while Dnak levels were detected as controls. The gray value calculated the half-life. (D) Acetylation of EspB enhances Tir into host cells via T3SS. HeLa cells were infected with wild-type EHEC or mutants containing Tir-TEM1 vectors. At $6 \mathrm{~h}$ of infection, cells were incubated with CCF2/AM1 dye. The $\beta$-lactamase activity in HeLa cells was detected by measuring the CCF2/AM substrate's cleavage and presented as the emission ratio of blue/green fluorescence $(460 / 530 \mathrm{~nm})$. White bars indicate $50 \mu \mathrm{m}$, data are represented as mean \pm SEM, and experiments shown were repeated at least two times. T3SS, type III secretion system; IPTG, isopropyl $\beta$-D-1-thiogalactopyranoside; EHEC, enterohemorrhagic Escherichia coli. 
the emission ratio of $460 / 530 \mathrm{~nm}$. It was shown that when ataT was absent, the emission ratio of $460 / 530 \mathrm{~nm}$ (blue/green) was reduced to about $77 \%$ compared with the WT or $\triangle a t a R$ groups (Figure 3D). Therefore, we concluded that AtaT acetylates EspB to maintain its stability, further enhancing the translocation of adhesion protein Tir into host cells.

\section{Acetylation of K206 Enhances EspB Stability and Modulates the Virulence of Enterohemorrhagic Escherichia coli}

In order to determine the modification sites of AtaT acetylated EspB, two EspB protein samples were analyzed by mass spectrometry. One was $6 \mathrm{xHis}-\mathrm{EspB}$ expressed from strain E. coli BL21(DE3) carrying the vector pET-EspB(his) ${ }_{6}$, and the other one was the above vector co-expressed with vector pBAD-AtaT. Of the 23 lysine residues in EspB, we identified six lysines (K47, K58, K92, K178, K192, and K206) by liquid chromatography-tandem mass spectroscopy (LC-MS/MS) analysis (Supplementary Table 2). We used I-TASTER to predict the tertiary structure of EspB. We found that all six acylated lysine residues were located at the exposed sites; K47, K58, and K92 were in the extracellular domain; and K178, K192, and K206 were in the intracellular domain (Figure 4A). Among them, K206 was the most significant acetylation site when comparing the ratio of acetylated peptides with unmodified peptides (Figure 4C and Supplementary Table 2).

To determine which sites are tightly associated with protein stability, these sites were mutated to alanine (A), glutamine (Q), or arginine (R), to mimic the non-acetylated, constitutively acetylated, and cannot be acetylated or deacetylated forms, respectively (Ren et al., 2016). We constructed these mutant expression vectors of EspB with six histidine tags at its $\mathrm{C}$ termini and tested these proteins' stability in EHEC. The results showed that the half-life of K206Q was approximately 1.4-fold longer than that of EspB WT, while the stability of K206R and K206A was slightly reduced (Figure 5A). Changes in acetyl levels at other sites were either independent of stability or inconsistent with the change of EspB WT modified by AtaT (Supplementary Figure 4). Modification on these sites may affect the basic functions of EspB or be redundantly modified. Importantly, K206 is located in the predicted protein-protein interaction domains of EspB (Luo and Donnenberg, 2011) and is highly conserved in bacteria (Figure 4B). Therefore, we suspect that EspB maintains its stability mainly via acetylation at the K206 site by AtaT.

Furthermore, to examine the effect of acetylated K206 on EHEC virulence, we infected HeLa cells with EHEC harboring EspB WT or EspBK206 derivatives. As expected, K206Q or EspB
A

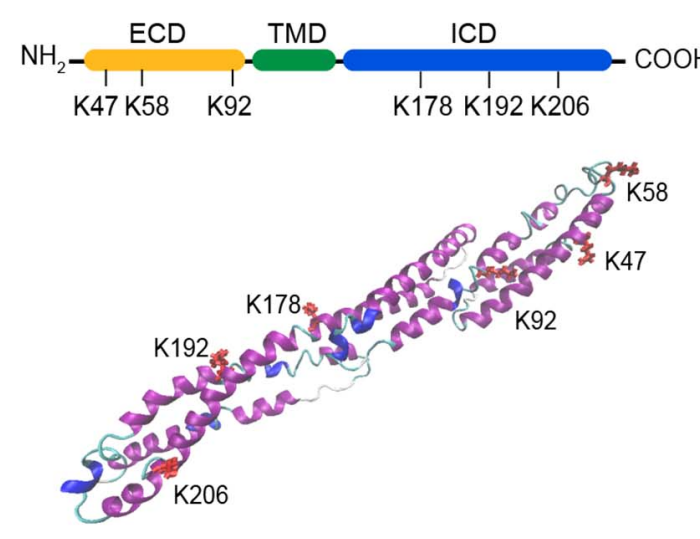

C

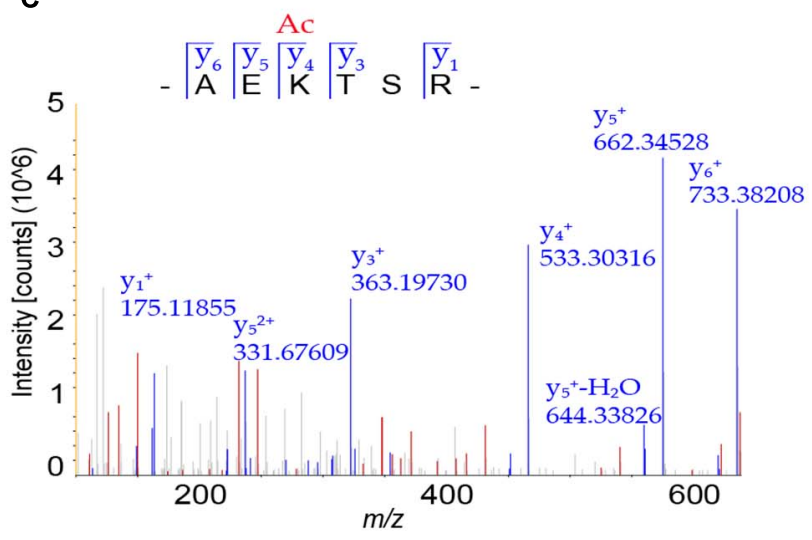

B

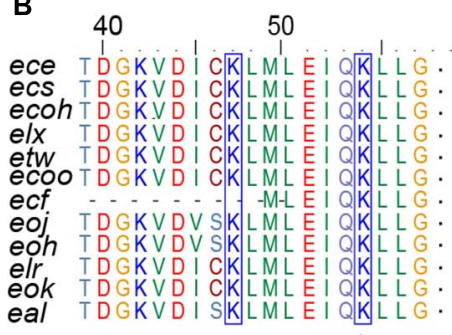

*

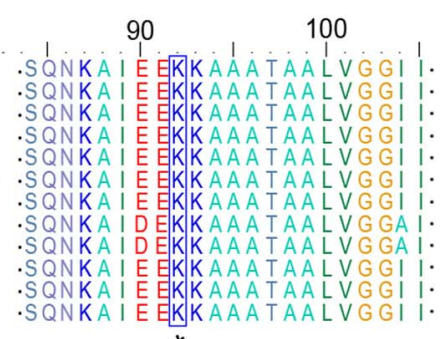

$*$

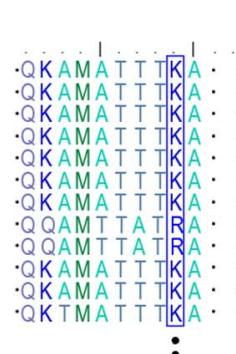

:

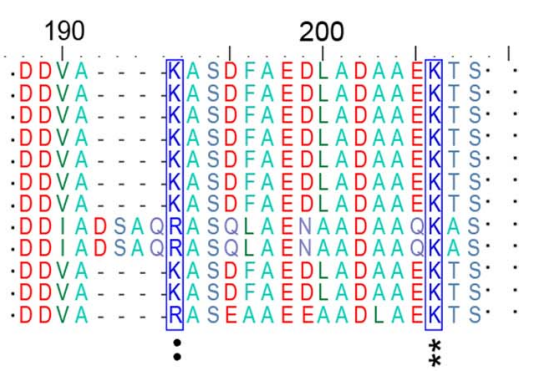

FIGURE 4 | Analysis of acetylation sites modified by AtaT in EspB. (A) Acetylation sites in domain architecture (top) and predicted tertiary structure of EspB (bottom). Structure prediction was performed with I-TASSER. ECD, extracellular domain, yellow. ICD, intracellular domain, green. TMD, transmembrane domain, blue. Lysine residues detected to be acylated (K47, K58, K92, K178, K192, and K206) are highlighted in red. (B) Conservation analysis of EspB lysine residues (blue box) through sequence alignment. Star, conserved; colon, conservative change; and the result was analyzed by BioEdit. (C) MS/MS spectrum of EspB K206 acetylated (K206Ac) peptide detected from EspB co-expression with AtaT in Escherichia coli BL21(DE)3. MS, mass spectroscopy. 
WT carrying strains showed higher adhesion ability compared with K206R or K206A at $6 \mathrm{~h}$ of infection (Figures 5B,C), and there were also similar trends at other infection time points. The IL-8 levels also showed that host inflammatory responses in the K206R and K206A groups were less than those in the K206Q or EspB WT strains groups, which is consistent with the trend of adhesion (Figure 5D). The above results showed that EspB K206Q mimicking acetylation could significantly increase the virulence of EHEC.

\section{DISCUSSION}

Type II TA is the most deeply studied TA, and it is involved in a variety of cell physiological activities. Although the role of type II TAs in the formation of persister cells has been questioned in recent years (Goormaghtigh et al., 2018a; Pontes and Groisman, 2019; Rosendahl et al., 2020), other functions, such as growth diminution during stress, biofilm formation, and phage inhibition, are still widely recognized (Wen et al., 2014; Sauert et al., 2016; Hosseini et al., 2019; Jurënas and Van Melderen, 2020; Song and Wood, 2020). In this work, we demonstrated that the type II toxins AtaT is involved in the virulence of EHEC, including strains colonization, inducing severe pathological injury and host inflammatory response.

ataRT, locus Z4833-Z4832 in EHEC O157:H7 EDL933, which the toxin AtaT of the AtaRT, is a member of the GNAT family. Blast analyses online show that AtaRT is distributed in various pathogens, such as Escherichia, Shigella, Klebsiella, and Salmonella (Supplementary Figure 1A). The strain can also present a basal growth state in the
A

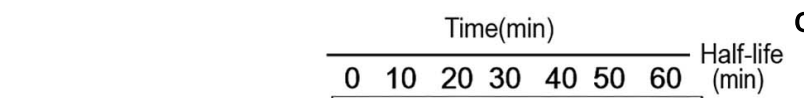

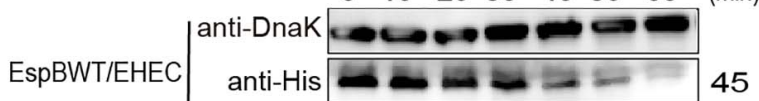

$1.01 .00 .80 .8 \quad 0.50 .4 \quad 0.1$

EspBK206A/EHEC $\mid \begin{aligned} & \text { anti-DnaK } \\ & \text { anti-His }\end{aligned}$

$\begin{array}{lllllllll}1.0 & 1.0 & 0.6 & 0.7 & 0.4 & 0.3 & 0.2\end{array}$

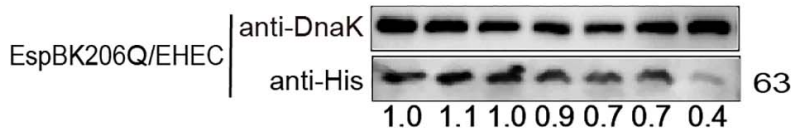

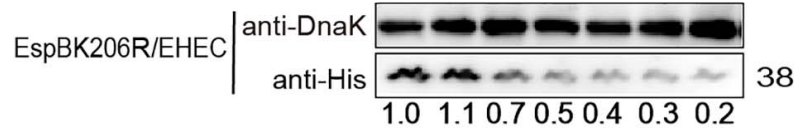

C
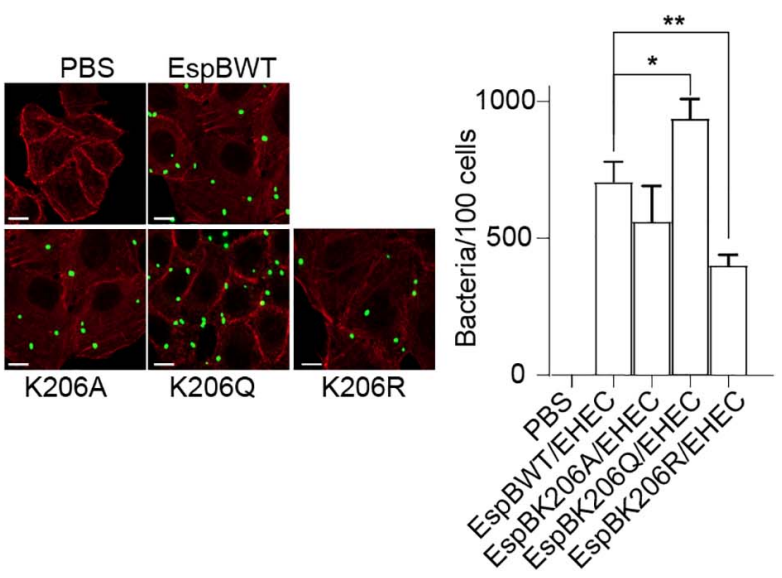

B

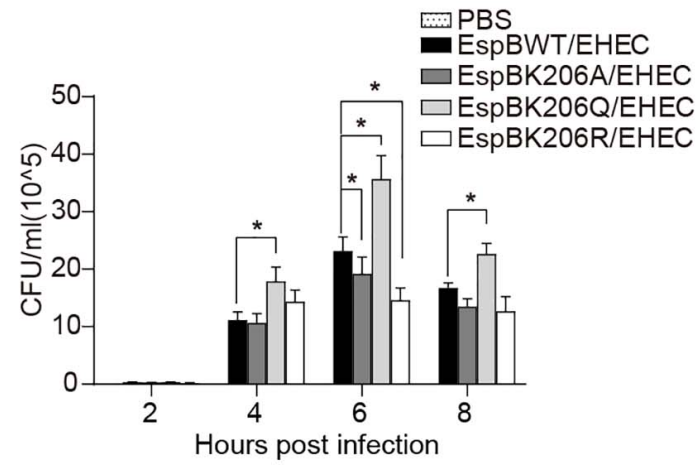

D

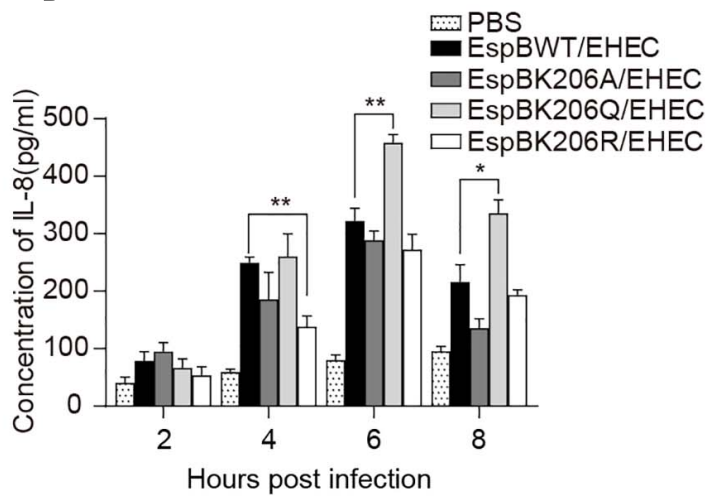

FIGURE 5 | Acetylation of EspB K206 enhances protein stability and promote the virulence of strains. (A) Stabilities of EspB WT, K206Q, K206R, and K206A in EHEC. Strains harboring EspB expression plasmid were induced by IPTG for $1 \mathrm{~h}$. Spectinomycin was used to terminate protein synthesis at different points and strains were collected. Levels of EspB were determined by anti-His antibody, while Dnak levels were detected as controls. The half-lives were calculated by the gray value. (B) EspB K206Q enhances EHEC adhesion ability to host cells. HT-29 cells were infected with EHEC harboring EspB wild-type or its derivatives at an MOI of 10. The gradient diluted cell lysates were inoculated on agar plates, and bacterial colonies were counted to calculate adhesion efficiency. (C) The number of bacteria attached after cells were infected for $6 \mathrm{~h}$ was observed under the fluorescence microscope. (D) IL-8 expression levels of host cells in different infection hours were determined by ELISA. Error bars represent SEM from $\geq 2$ independent experiments, ${ }^{*} P<0.05,{ }^{\star *} P<0.01$ compared with EspB WT/EHEC. WT, wild-type; IPTG, isopropyl $\beta$-D-1-thiogalactopyranoside; ELISA, enzyme-linked immunosorbent assay; IL-8, interleukin 8; EHEC, enterohemorrhagic Escherichia coli; MOI, multiplicity of infection. 
absence of cognate antitoxin (Supplementary Figure 1B), suggesting that AtaT shows "weaker" toxicity. It was inferred that AtaT may have other non-toxic functions. In this study, ataRT (especially ataT) expression was upregulated during strain infection, and AtaT promotes EHEC virulence (Figures 1, 2). Although we do not know which infection signal triggered the TAs, these data provide strong evidence that the GNAT toxin AtaT is involved in regulating strains' pathogenicity.

Adhesion plays a vital role in host colonization of strains. EHEC contains a battery of adhesion proteins, including intimin, Tir, Tccp, T3SS translocons (EspA, EspB, and EspD), and Lpf (long polar fimbria) (McWilliams and Torres, 2014). Many virulence proteins (effectors) are translocated into host cells via T3SS (Bohn et al., 2019), and EspB is the base element built on the host cell. Notably, AtaT can specifically acetylate EspB directly and promote its stability, so the adhesion proteins, such as Tir, can be efficiently transported into host cells, facilitating close attachment of the bacteria (Figure 3). Except for TacT, which can acetylate both TacA and tRNAs synchronously in Salmonella, no known GNAT toxin has been found to acetylate proteins (VanDrisse et al., 2017). Jurenas et al. (2017) used isotope-labeled $\left({ }^{14} \mathrm{C}\right)$ Ac CoA to monitor the acetylation reaction catalyzed by the AtaT in vitro translation reaction. The product was dissolved on SDS-PAGE gels, and no signal was detected, so they suggested that either the target was not a protein or the acetylation was unstable in this condition. In our co-expression reaction in $E$. coli BL21(DE)3, we revealed an apparent increase in the degree of acetylation. This is the first report that AtaT can directly modify protein besides tRNA.

EspB maintains its stability mainly via the acetylation of K206. However, we do not know how the change in the acetylation state of EspB K206 regulates its stability. Recently, Eshun-Wilson et al. (2019) reported that acetylation improved $\alpha$-tubulin stability and explained the structural mechanism. They showed that deacetylated $\mathrm{K} 40$ in an $\alpha 1$-monomer is close to the M-loop, supporting lateral interaction. When $\mathrm{K} 40$ is acetylated, it packs $\sim 10 \AA$ closer to the globular domain, reducing the potential for intermonomer interactions. Data from another study (Song et al., 2019) showed that the deacetylation-mimic in K49 and K51 had a larger surface area than the acetylation-mimic. As a large surface area is often required for potent protein interactions, acetylation might decrease the binding affinity of LC3 family proteins to its potential interacting protein. As mentioned in the results, K206 is located in the predicted protein-protein interaction domains of EspB. We propose that the acetylation of K206 may alter the conformational landscape. Less surface area blocks EspP or other interacting proteins from approaching it, thereby hindering the degradation of acetylated EspB.

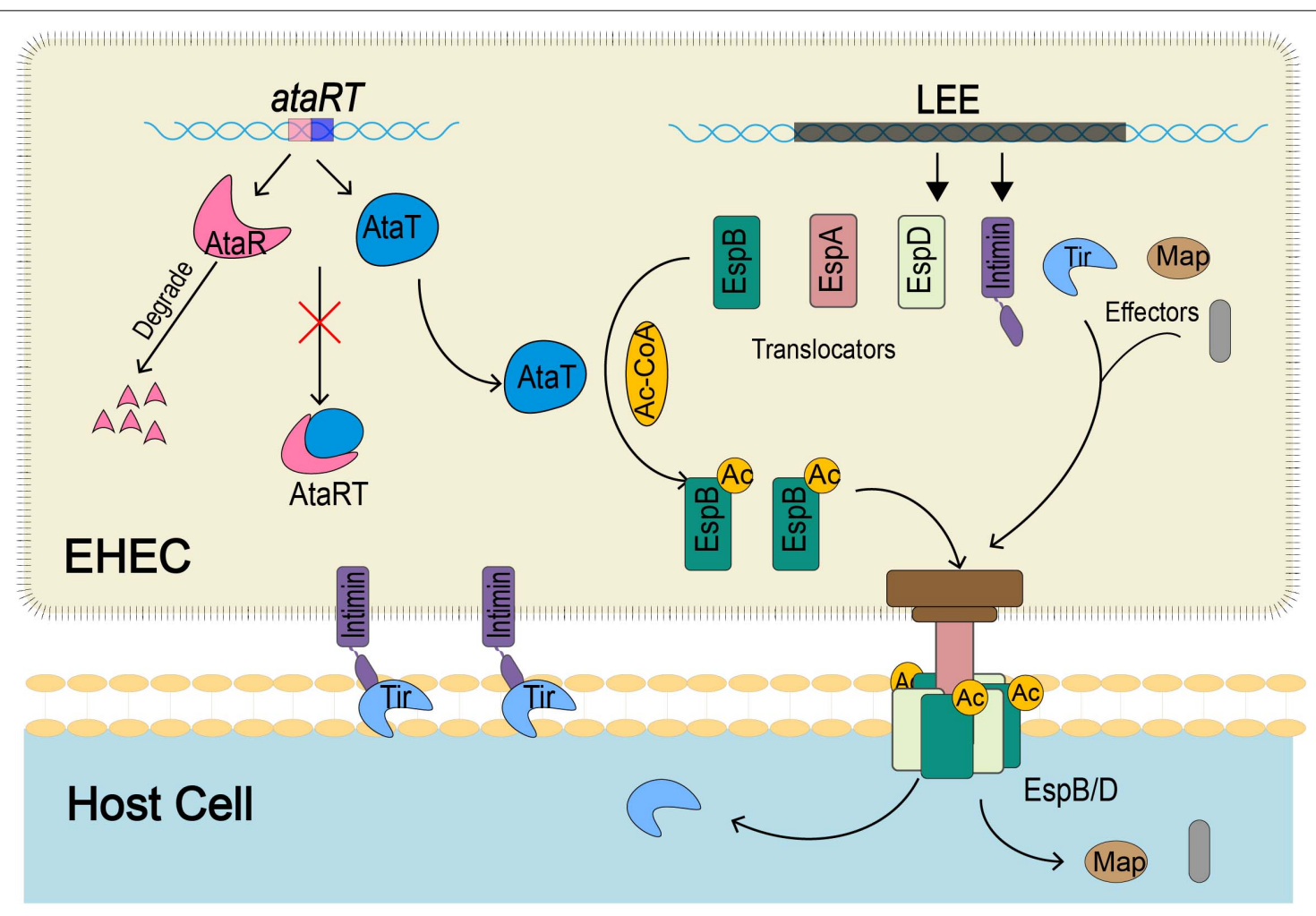

FIGURE 6 | The schematic diagram of how EspB acetylated modification by AtaT enhances EHEC virulence. During host infection by strains, AtaT acetylates the EspBK206 site with acetyl-CoA, ensuring that EspB can be secreted extracellularly to form a stable T3SS base with EspD, enhancing early adhesion. Then Tir and other effectors transported into the host cell via T3SS efficiently cause tight attachment, host inflammatory responses, and tissue injury. T3SS, type III secretion system; EHEC, enterohemorrhagic Escherichia coli. 
Notably, AtaT and EspB are both widely present in EHEC and enteropathogenic E. coli (EPEC) (data not shown). In addition, the EspB modification site K206 is very conserved in the strain distribution (Figure 4), indicating that the model of AtaT modifying EspB is probably a comprehensive regulation. If we find molecules that can intervene or reverse K206 modification by AtaT, we may be able to deal with most of the pathogenic problems of EHEC and EPEC.

\section{CONCLUSION}

In summary, our study revealed that the GANT toxin AtaT could enhance the virulence of strains and explained the mechanism (Figure 6). ataRT genes upregulate during infection, and AtaT acetylates EspB with acetyl-CoA. The modified EspB is secreted extracellularly, forms a channel complex with EspD, and then stably embedded in the host cell membrane. Then effectors such as Tir are transported into the host cell efficiently, where they cause tight attachment, host inflammatory responses, and tissue injury.

\section{DATA AVAILABILITY STATEMENT}

The raw data supporting the conclusions of this article will be made available by the authors, without undue reservation.

\section{REFERENCES}

Alsharif, G., Ahmad, S., Islam, M. S., Shah, R., Busby, S. J., and Krachler, A. M. (2015). Host attachment and fluid shear are integrated into a mechanical signal regulating virulence in Escherichia coli O157:H7. Proc. Natl. Acad. Sci. U.S.A. 112, 5503-5508. doi: 10.1073/pnas.1422986112

Bohn, E., Sonnabend, M., Klein, K., and Autenrieth, I. B. (2019). Bacterial adhesion and host cell factors leading to effector protein injection by type III secretion system. Int. J. Med. Microbiol. 309, 344-350. doi: 10.1016/j.ijmm.2019. 05.008

Bravo, A., de Torrontegui, G., and Díaz, R. (1987). Identification of components of a new stability system of plasmid R1, ParD, that is close to the origin of replication of this plasmid. Mol. Gen. Genet. 210, 101-110. doi: 10.1007/ bf00337764

Cameron, E. A., Curtis, M. M., Kumar, A., Dunny, G. M., and Sperandio, V. (2018). Microbiota and pathogen proteases modulate type III secretion activity in Enterohemorrhagic Escherichia coli. mBio 9, e02204-18. doi: 10.1128/mBio. 02204- 18

Cheverton, A. M., Gollan, B., Przydacz, M., Wong, C. T., Mylona, A., Hare, S. A., et al. (2016). A Salmonella toxin promotes persister formation through acetylation of tRNA. Mol. Cell 63, 86-96. doi: 10.1016/j.molcel.2016. 05.002

Eshun-Wilson, L., Zhang, R., Portran, D., Nachury, M. V., Toso, D. B., Löhr, T., et al. (2019). Effects of $\alpha$-tubulin acetylation on microtubule structure and stability. Proc. Natl. Acad. Sci. U.S.A. 116, 10366-10371. doi: 10.1073/pnas. 1900441116

Fraikin, N., Goormaghtigh, F., and Van Melderen, L. (2020). Type II toxinantitoxin systems: evolution and revolutions. J. Bacteriol. 202, e00763-19. doi: 10.1128/JB.e00763-19

Gerdes, K., Christensen, S. K., and Lobner-Olesen, A. (2005). Prokaryotic toxinantitoxin stress response loci. Nat. Rev. Microbiol. 3, 371-382. doi: 10.1038/ nrmicro1147

\section{ETHICS STATEMENT}

The animal study was reviewed and approved by the Institutional Ethics Review Committee of Beijing Institute of Microbiology and Epidemiology, China.

\section{AUTHOR CONTRIBUTIONS}

$\mathrm{ZH}$ performed the experiments, analyzed the data, and wrote the manuscript. TL, JW, ZL, DL, and NN analyzed the data. FC and ZL performed the experiments. HW designed this study, analyzed the data, wrote the manuscript, and supervised the project. All authors discussed the results and commented on the manuscript.

\section{FUNDING}

This work was supported by the National Key Basic Research Program (973) of China (2015CB554202) and the Beijing Nova Program (Z181100006218111).

\section{SUPPLEMENTARY MATERIAL}

The Supplementary Material for this article can be found online at: https://www.frontiersin.org/articles/10.3389/fmicb. 2021.627141/full\#supplementary-material

Gerdes, K., Rasmussen, P. B., and Molin, S. (1986). Unique type of plasmid maintenance function: postsegregational killing of plasmid-free cells. Proc. Natl. Acad. Sci. U.S.A. 83, 3116-3120. doi: 10.1073/pnas.83.10. 3116

Goormaghtigh, F., Fraikin, N., Putrins, M., Hauryliuk, V., Garcia-Pino, A., Udekwu, K., et al. (2018a). Reply to holden and errington, "Type II toxinantitoxin systems and persister cells". mBio 9:e01838-18. doi: 10.1128/mBio. 01838-18

Goormaghtigh, F., Fraikin, N., Putrinš, M., Hallaert, T., Hauryliuk, V., GarciaPino, A., et al. (2018b). Reassessing the role of type II toxin-antitoxin systems in formation of Escherichia coli type II persister cells. mBio 9:e00640-18. doi: 10.1128/mbio.00640-18

Harms, A., Brodersen, D. E., Mitarai, N., and Gerdes, K. (2018). Toxins, targets, and triggers: an overview of toxin-antitoxin biology. Mol. Cell 70, 768-784. doi: 10.1016/j.molcel.2018.01.003

Holden, D. W., and Errington, J. (2018). Type II toxin-antitoxin systems and persister cells. $m$ Bio 9, e01574-18. doi: 10.1128/mBio.01574-18

Hosseini, N., Pourhajibagher, M., Chiniforush, N., Hosseinkhan, N., Rezaie, P., and Bahador, A. (2019). Modulation of toxin-antitoxin system Rnl AB Type II in phage-resistant Gammaproteobacteria surviving photodynamic treatment. J. Lasers Med. Sci. 10, 21-28. doi: 10.15171/jlms.2019.03

Jurenas, D., Chatterjee, S., Konijnenberg, A., Sobott, F., Droogmans, L., GarciaPino, A., et al. (2017). AtaT blocks translation initiation by $\mathrm{N}$-acetylation of the initiator tRNA(fMet). Nat. Chem. Biol. 13, 640-646. doi: 10.1038/nchembio. 2346

Jurënas, D., and Van Melderen, L. (2020). The variety in the common theme of translation inhibition by Type II toxin-antitoxin systems. Front. Genet. 11:262. doi: $10.3389 /$ fgene.2020.00262

Lang, C., Fruth, A., Holland, G., Laue, M., Muhlen, S., Dersch, P., et al. (2018). Novel type of pilus associated with a Shiga-toxigenic E. coli hybrid pathovar conveys aggregative adherence and bacterial virulence. Emerg. Microbes Infect. 7:203. doi: 10.1038/s41426-018-0209-8 
Li, T., Li, Z., Chen, F., Liu, X., Ning, N., Huang, J., et al. (2017). Eukaryoticlike kinase expression in enterohemorrhagic Escherichia coli: potential for enhancing host aggressive inflammatory response. J. Infect. Dis. 216, 11501158. doi: 10.1093/infdis/jix160

Lobato-Marquez, D., Diaz-Orejas, R., and Garcia-Del Portillo, F. (2016). Toxinantitoxins and bacterial virulence. FEMS Microbiol. Rev. 40, 592-609. doi: 10 . 1093/femsre/fuw022

Luo, W., and Donnenberg, M. S. (2006). Analysis of the function of enteropathogenic Escherichia coli EspB by random mutagenesis. Infect. Immun. 74, 810-820. doi: 10.1128/iai.74.2.810-820.2006

Luo, W., and Donnenberg, M. S. (2011). Interactions and predicted host membrane topology of the enteropathogenic Escherichia coli translocator protein EspB. J. Bacteriol. 193, 2972-2980. doi: 10.1128/jb.00153-11

McVicker, G., and Tang, C. M. (2016). Deletion of toxin-antitoxin systems in the evolution of Shigella sonnei as a host-adapted pathogen. Nat. Microbiol. 2:16204. doi: 10.1038/nmicrobiol.2016.204

McWilliams, B. D., and Torres, A. G. (2014). Enterohemorrhagic Escherichia coli adhesins. Microbiol. Spectr. 2:3. doi: 10.1128/microbiolspec.EHEC-0003-2013

Mills, E., Baruch, K., Aviv, G., Nitzan, M., and Rosenshine, I. (2013). Dynamics of the type III secretion system activity of enteropathogenic Escherichia coli. mBio 4, e00303-13. doi: 10.1128/mBio.00303-13

Ogura, T., and Hiraga, S. (1983). Mini-F plasmid genes that couple host cell division to plasmid proliferation. Proc. Natl. Acad. Sci. U.S.A. 80, 4784-4788. doi: $10.1073 /$ pnas.80.15.4784

Paul, P., Sahu, B. R., and Suar, M. (2019). Plausible role of bacterial toxin-antitoxin system in persister cell formation and elimination. Mol Oral Microbiol 34, 97-107. doi: 10.1111/omi.12258

Pontes, M. H., and Groisman, E. A. (2019). Slow growth determines nonheritable antibiotic resistance in Salmonella enterica. Sci Signal 12:eaax3938. doi: 10.1126/ scisignal.aax3938

Qian, H., Yao, Q., Tai, C., Deng, Z., Gan, J., and Ou, H. Y. (2018). Identification and characterization of acetyltransferase-type toxin-antitoxin locus in Klebsiella pneumoniae. Mol. Microbiol. 108, 336-349. doi: 10.1111/mmi.13934

Qian, H., Yu, H., Li, P., Zhu, E., Yao, Q., Tai, C., et al. (2019). Toxinantitoxin operon kacAT of Klebsiella pneumoniae is regulated by conditional cooperativity via a W-shaped KacA-KacT complex. Nucleic Acids Res. 47, 7690-7702. doi: 10.1093/nar/gkz563

Ren, J., Sang, Y., Tan, Y., Tao, J., Ni, J., Liu, S., et al. (2016). Acetylation of lysine 201 inhibits the DNA-binding ability of PhoP to regulate Salmonella virulence. PLoS Pathog. 12:e1005458. doi: 10.1371/journal.ppat.1005458

Riffaud, C., Pinel-Marie, M. L., and Felden, B. (2020). Cross-Regulations between bacterial toxin-antitoxin systems: evidence of an interconnected regulatory network? Trends Microbiol. 28, 851-866. doi: 10.1016/j.tim.2020.05.016

Rocker, A., and Meinhart, A. (2016). Type II toxin: antitoxin systems. More than small selfish entities? Curr. Genet. 62, 287-290. doi: 10.1007/s00294-0150541-7

Rosendahl, S., Tamman, H., Brauer, A., Remm, M., and Horak, R. (2020). Chromosomal toxin-antitoxin systems in Pseudomonas putida are rather selfish than beneficial. Sci. Rep. 10:9230. doi: 10.1038/s41598-020-65504-0

Rycroft, J. A., Gollan, B., Grabe, G. J., Hall, A., Cheverton, A. M., Larrouy-Maumus, G., et al. (2018). Activity of acetyltransferase toxins involved in Salmonella persister formation during macrophage infection. Nat. Commun. 9:1993. doi: 10.1038/s41467-018-04472-6

Sang, Y., Ren, J., Ni, J., Tao, J., Lu, J., and Yao, Y. F. (2016). Protein acetylation is involved in Salmonella enterica serovar typhimurium virulence. J. Infect. Dis. 213, 1836-1845. doi: 10.1093/infdis/jiw028
Sauert, M., Wolfinger, M. T., Vesper, O., Muller, C., Byrgazov, K., and Moll, I. (2016). The MazF-regulon: a toolbox for the post-transcriptional stress response in Escherichia coli. Nucleic Acids Res. 44, 6660-6675. doi: 10.1093/nar/gkw115

Song, S., and Wood, T. K. (2020). Toxin/Antitoxin system paradigms: toxins bound to antitoxins are not likely activated by preferential antitoxin degradation. $A d v$. Biosyst. 4:e1900290. doi: 10.1002/adbi.201900290

Song, T., Su, H., Yin, W., Wang, L., and Huang, R. (2019). Acetylation modulates LC3 stability and cargo recognition. FEBS Lett. 593, 414-422. doi: 10.1002/ 1873-3468.13327

Tsilibaris, V., Maenhaut-Michel, G., Mine, N., and Van Melderen, L. (2007). What is the benefit to Escherichia coli of having multiple toxin-antitoxin systems in its genome? J. Bacteriol. 189, 6101-6108. doi: 10.1128/JB.00527-07

Van Melderen, L., Jurenas, D., and Garcia-Pino, A. (2018). Messing up translation from the start: how AtaT inhibits translation initiation in E. coli. RNA Biol. 15, 303-307. doi: 10.1080/15476286.2017.1391439

Van Melderen, L., and Saavedra De Bast, M. (2009). Bacterial toxin-antitoxin systems: more than selfish entities? PLoS Genet. 5:e1000437. doi: 10.1371/ journal.pgen.1000437

VanDrisse, C. M., Parks, A. R., and Escalante-Semerena, J. C. (2017). A toxin involved in Salmonella persistence regulates its activity by acetylating its cognate antitoxin, a modification reversed by CobB sirtuin deacetylase. mBio 8, e00708-17. doi: 10.1128/mBio.00708-17

Walling, L. R., and Butler, J. S. (2019). Toxins targeting transfer RNAs: translation inhibition by bacterial toxin-antitoxin systems. Wiley Interdiscip. Rev. RNA 10:e1506. doi: 10.1002/wrna.1506

Wen, Y., Behiels, E., and Devreese, B. (2014). Toxin-Antitoxin systems: their role in persistence, biofilm formation, and pathogenicity. Pathog. Dis. 70, 240-249. doi: 10.1111/2049-632X.12145

Wilcox, B., Osterman, I., Serebryakova, M., Lukyanov, D., Komarova, E., Gollan, B., et al. (2018). Escherichia coli ItaT is a type II toxin that inhibits translation by acetylating isoleucyl-tRNAIle. Nucleic Acids Res. 46, 7873-7885. doi: 10.1093/ nar/gky560

Xie, Y., Wei, Y., Shen, Y., Li, X., Zhou, H., Tai, C., et al. (2018). TADB 2.0: an updated database of bacterial type II toxin-antitoxin loci. Nucleic Acids Res. 46, D749-D753. doi: 10.1093/nar/gkx1033

Yamaguchi, Y., and Inouye, M. (2011). Regulation of growth and death in Escherichia coli by toxin-antitoxin systems. Nat. Rev. Microbiol. 9, 779-790. doi: 10.1038/nrmicro2651

Yashiro, Y., Yamashita, S., and Tomita, K. (2019). Crystal structure of the enterohemorrhagic Escherichia coli AtaT-AtaR Toxin-antitoxin complex. Structure 27, 476-484 e473. doi: 10.1016/j.str.2018.11.005

Yeo, C. C. (2018). GNAT toxins of bacterial toxin-antitoxin systems: acetylation of charged tRNAs to inhibit translation. Mol. Microbiol. 108, 331-335. doi: $10.1111 / \mathrm{mmi} .13958$

Conflict of Interest: The authors declare that the research was conducted in the absence of any commercial or financial relationships that could be construed as a potential conflict of interest.

Copyright (C) $2021 \mathrm{He}$, Li, Wang, Luo, Ning, Li, Chen and Wang. This is an openaccess article distributed under the terms of the Creative Commons Attribution License (CC BY). The use, distribution or reproduction in other forums is permitted, provided the original author(s) and the copyright owner(s) are credited and that the original publication in this journal is cited, in accordance with accepted academic practice. No use, distribution or reproduction is permitted which does not comply with these terms. 